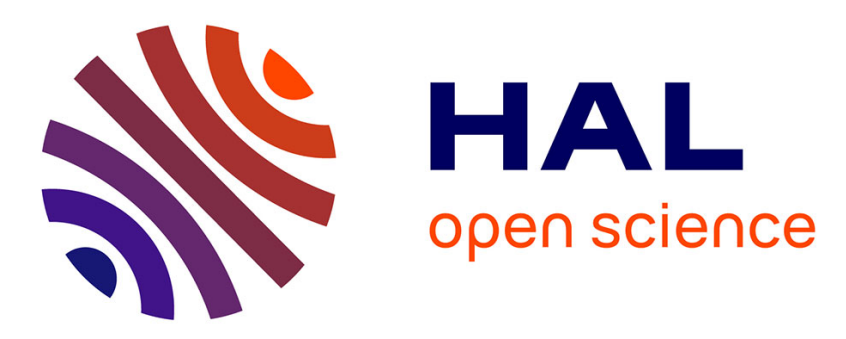

\title{
Refractive index determination in diamond anvil cells: Results for argon
}

\author{
M Grimsditch, R Letoullec, A. Polian, Michel Gauthier
}

\section{To cite this version:}

M Grimsditch, R Letoullec, A. Polian, Michel Gauthier. Refractive index determination in diamond anvil cells: Results for argon. Journal of Applied Physics, 1986. hal-03059424

\section{HAL Id: hal-03059424 \\ https://hal.science/hal-03059424}

Submitted on 12 Dec 2020

HAL is a multi-disciplinary open access archive for the deposit and dissemination of scientific research documents, whether they are published or not. The documents may come from teaching and research institutions in France or abroad, or from public or private research centers.
L'archive ouverte pluridisciplinaire HAL, est destinée au dépôt et à la diffusion de documents scientifiques de niveau recherche, publiés ou non, émanant des établissements d'enseignement et de recherche français ou étrangers, des laboratoires publics ou privés. 


\title{
Refractive index determination in diamond anvill cells: Results for argon
}

\author{
M. Grimsditch \\ Materials Science Division, Argonne National Laboratory, Argonne, Illinois 60439 \\ R. Letoullec, A. Polian, and M. Gauthier \\ Laboratoire Physique des Milieux Condensés (C.N.R.S.-U.A. 782), Université P. et M. Curie T13-E4, \\ 4 Place Jussieu, F-75252 Paris Cedex 05
}

(Received 18 June 1986; accepted for publication 5 August 1986)

The pressure dependence of the refractive index of argon has been measured in a diamond anvil cell up to $15 \mathrm{GPa}$. We describe in detail the novel technique used, which offers considerably higher accuracy than the others previously utilized. Our results on Ar agree with previous results at lower pressures and are compared with theoretical calculations at high pressure.

\section{INTRODUCTION}

The advent of the diamond anvil cell (DAC) has revolutionized high-pressure studies by adding almost two orders of magnitude to the static pressures which can now be routinely achieved in the laboratory. ${ }^{1}$ However, due to the small size of the sample $(\sim 1 \mu \mathrm{g})$, many of the conventional tools used for material characterization are not applicable. It is then of general interest to develop methods which allow different properties to be measured: here we shall deal with the determination of the refractive index $n$.

The determination of the refractive index is of interest since it is directly related to more fundamental properties such as the density and band structure. Furthermore, it is of indirect interest in Brillouin scattering experiments, where it is required in the data analysis and also from the technical side if the distance between the diamond anvils is to be determined.

The most accurate method to determine $n$ is that used for GaS (Ref. 2) and GaP (Ref. 3). This method can be used in cases where the sample can be placed in the cell in the form of a thin platelet. The faces of the sample act as a FabryPérot interferometer which produces interferences in the transmitted light. From the distance between the successive transmission maxima or minima, the product $n d$ can be determined ( $d$ is the sample thickness). If the compressibility and the thickness at zero pressure are known, $n$ can be evaluated.

A second method which can be used for samples in the platelet geometry and of known orientation is Brillouin scattering, ${ }^{4}$ using two different scattering geometries it involves the determination of the sound velocity $v$ and the product $n v$. Provided the experiments probe the same propagation direction (and consequently the same sound velocity), $n$ can be determined.

Both above methods fail when dealing with samples that are loaded into the cell as a fluid and which crystallize randomly when pressure is increased. In the first method, even though interferences fringes are still observed between the diamond surfaces, the distance $d$ can now no longer be estimated in a straightforward manner. The second method fails since different velocities are measured in the two scattering geometries.
In principle, if a series of samples of different refractive indices could be measured by either of the two above-mentioned techniques, then the pressure at which index matching occurs between the sample and the surrounding fluid or solid could be used to determine $n$ of the later. However, even though this was proven to be a reliable method, ${ }^{5}$ it is difficult (or even impossible as in the case of helium) to find the adequate set of reference samples.

The method which has been used to date for samples that crystallize in the cell completely filling the space between the diamonds relies on the fact that the anvils act as a Fabry-Perot interferometer so that a maximum in transmitted light occurs when

$$
\text { 2nd } \cos \phi=K \lambda \text {, }
$$

where $K$ is an integer (interference order), $\lambda$ the wavelength, $d$ the distance between the diamonds, and $\phi$ the angle inside the cell that the light makes with the cell axis. After accounting for refraction, Eq. (1) can be written in terms of $\theta$, the angle the light makes with the cell normal outside the cell, viz.

$$
2 n d\left(1-\frac{\sin ^{2} \theta}{n^{2}}\right)^{1 / 2}=K \lambda
$$

Typically, a transmission curve (Fig. 1) as a function of wavelength is recorded. Then, by rotating the cell to change $\theta$, a similar spectrum is recorded, and from the shift in the

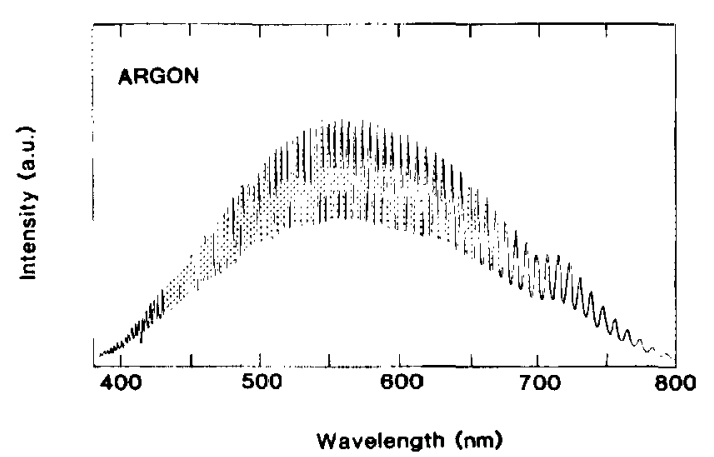

FIG. 1. Typical interference spectrum of argon in the DAC at $2.9 \mathrm{GPa}$. The shape of the envelope curves results from the convolution of the spectral distribution of the quartz-iodine lamp with the response of the spectrometer. 
transmission maxima the distance $d$ and $n$ can both be calculated. ${ }^{6}$ This conceptually simple method suffers from a serious drawback: it requires that either $d$ be a constant (perfectly parallel diamonds) or that exactly the same spot be probed after the cell has been rotated. Since neither of these conditions is easy to satisfy, the reported results contain errors as large as $30 \%$ in $n$.

\section{DESCRIPTION OF THE METHOD}

In order to overcome the above difficulties, we use the following method: in a first step, we determine the interference order $K$ for $\theta=0$ from a spectrum as shown in Fig. 1; in a second step we use a beam of monochromatic light focused in the cell with a very wide aperture lens. It is then possible to probe different angles without moving the cell, thus making sure that the same spot is being probed at all angles. The setup we have used is shown in Fig. 2. The purpose of the elements in our setup are as follows: The combination of the pinhole $P$ and the lenses $L 1$ and $L 2$ allows the full solid angle of the cell to be utilized, the image of $P$ being focused onto the hole in the gasket.

If, with the setup of Fig. 2, the light transmitted through the cell is allowed to fall on a screen, a set of concentric rings is observed as given by Eq. (2). The intensity distribution as a function of angle is schematically shown in Fig. 3(a), and the depth of the troughs is proportional to the square of the difference between the refractive indices of diamond and the material in the cell. This translates into the fact that when $n>1.3$ the fringes become unobservable to the naked eye. To improve the contrast, the fringes can be observed in reflection by placing a beam splitter $S$ between the cell and the lens $L 2$. Unfortunately, the light reflected at the front surface of the diamond produces a very intense background so that only an intensity distribution shown in Fig. 3(b) is obtained and not much is gained compared with the observation in transmission. The final arrangement shown in Fig. 2 spatially filters out the radiation from the front surface of the diamond, and fringes of excellent contrast [Fig. 3(c)] can be observed on a screen placed as shown.

The angle $\theta$ can be calculated from the fringes by measuring their diameter and the distance from the pinhole to the screen if the magnification produced by the lens $L 2$ is known. (The simplest method to calibrate the system is with an empty cell, i.e., $n=1$.) If the angles subtended by the fringes are $\theta_{i}$ (numbering starts at the center) and $K_{i}$ is the

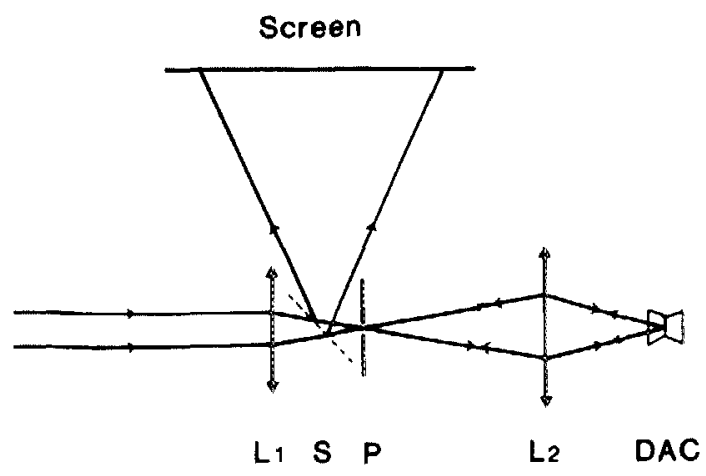

FIG. 2. Experimental setup: $L_{1}$ and $L_{2}$ lenses, $S$ beam splitter, $P$ pinhole.

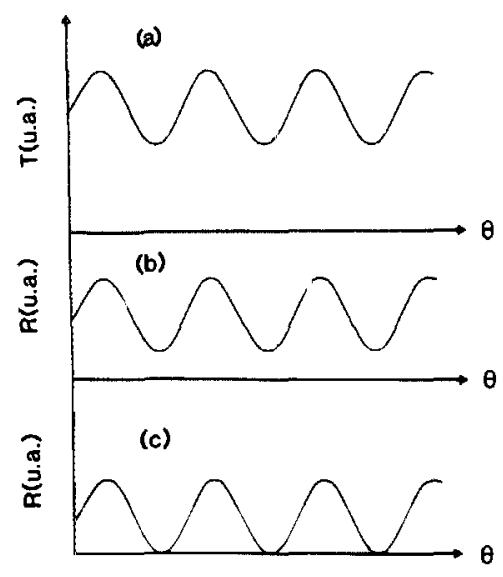

FIG. 3. (a) Angular distribution of the light transmitted through the DAC with the setup of Fig. 2. (b) Angular distribution of the reflected light when the pinhole of the setup of Fig. 2 is removed. (c) Actual angular distribution of the light observed with the full setup of Fig. 2.

interference order of the $i$ th fringe, $n$ can be written [see Eq. (2)]:

$$
n^{2}=K_{j}^{2}\left(\sin ^{2} \theta_{j}-\sin ^{2} \theta_{i}\right) /\left(K_{i}^{2}-K_{j}^{2}\right)+\sin ^{2} \theta_{j}
$$

The interference order of the first ring can be determined exactly from a transmission curve as shown in Fig. 1, and since the interference order of successive rings decreases by unity, Eq. (3) can be used to calculate $n$.

\section{RESURTS FOR ARGON}

Since no material exists which crystallizes in the cell and for which the refractive index has been accurately measured at high pressures, we have chosen to study argon because it is optically isotropic and because its refractive index can be calculated from its simple electronic structure and known equation of state. ${ }^{5,7}$ This provides us with a check for our experimental results.

Our experiments were performed in a conventional Block-Piermarini cell ${ }^{8}$ with an aperture half angle of $15^{\circ}$. The cell was loaded with argon as described in Ref. 9. At low pressures and with a gasket thickness of $\sim 70 \mu \mathrm{m}$, we observed 5-6 fringes which enabled us to obtain $n$ with reasonable accuracy. At higher pressures, decreasing gasket thickness $(\sim 20 \mu \mathrm{m})$ reduced the number of observed fringes to 2 . In order to increase the accuracy (the errors will be discussed in detail in the next section) we managed to increase the number of observed rings by filling the conical aperture of the cell with a liquid of high refractive index. This technique, which increases the effective aperture by $n$ of the liquid, allowed us to observe 3-4 fringes at $15 \mathrm{GPa}$.

Our experimental results are shown in Fig. 4 (points). The error bars are based only on the scatter that arises when different pairs of fringes are used in $\mathrm{Eq}$. (3) to calculate $n$. The full line in Fig. 4 is the calculated refractive index 9 and the stars are the experimental results from Ref. 10. At low pressures ( $<1 \mathrm{GPa}$ ), our experimental results agree with the very accurate results from Ref. 10. Within the experimental scatter, our results are also in agreement with the calculations ${ }^{5}$ over the whole investigated pressure range. At higher pressures, however, the scatter in our values is larger 


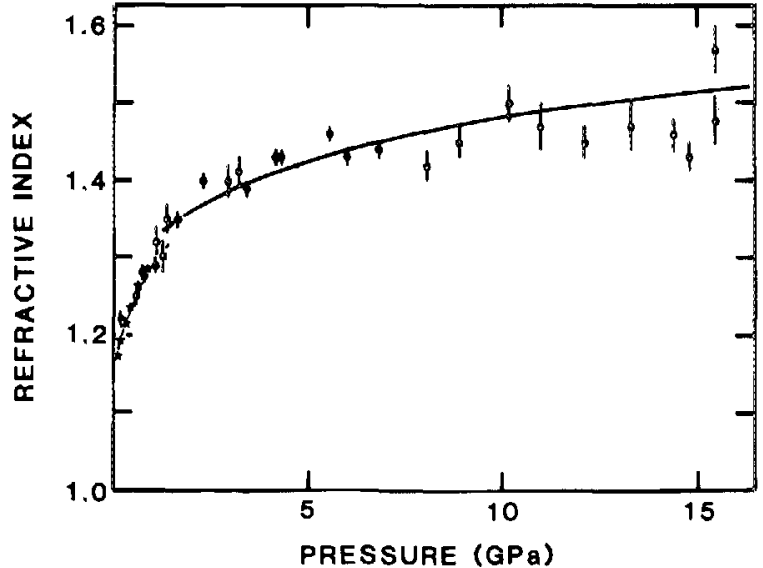

FIG. 4. Refractive index of argon vs pressure. The stars are results of Ref. 10; the continuous line is a theoretical result obtained from Ref. 9; the points are our results.

than the error estimated as described above, indicating that other sources of error must be present. In spite of this, the scatter in our results at $\sim 15 \mathrm{GPa}$ is around $4 \%$, which is to be compared with the $\sim 20 \%$ of previous attempts. ${ }^{6}$

\section{DISCUSSION OF ERRORS}

The only other term in Eq. (3) which can lead to errors is $K$. We have found however that from transmission measurements (Fig. 1) $\mathrm{K}$ can be determined exactly. Even allowing for possible nonparallelism of the diamonds, the resulting error in $K$ can be evaluated to be less than $1 \%$, which translates into an error of $\sim 0.5 \%$ in $n$. This statement was verified experimentally by purposely misaligning the diamonds: at low pressure the ring pattern is slightly deteriorated, but we obtain the same value for $n$. (We cannot go to high pressures with nonparallel diamonds). Since our values of $n$ calculated using Eq. (3) depend only on $\theta_{i}$ and $\mathrm{K}_{i}$, and the errors in the parameters do not account for the observed scatter, we are obliged to conclude that Eq. (3) must be at fault. Furthermore, since the only assumption leading to Eqs. (1) $-(3)$ is the presence of two flat surfaces, we must question whether this remains true at high pressures. That this may no longer be true has been proposed in Ref. 11 . We mention here that the method described in this paper has also allowed us to measure the curvature of the diamonds. A complete study of the deformation of the diamonds culets will be presented elsewhere. ${ }^{12}$ It is sufficient here to state that the radii of curvature are of the order of $10 \mathrm{~mm}$ at $\sim 15 \mathrm{GPa}$. The effect of this curvature on Eq. (3) is not straightforward to estimate, but it is likely to explain the observed scatter in our results, especially when coupled to the focusing or defocusing effect, that a curved surface will have on the position of the fringes.

\section{CONCLUSION}

We have presented a method for determining the refractive indices of materials in diamond anvil cells. The accuracy of the method is considerably better than that of previous determinations. Results for argon are presented and compared with theory and low-pressure results.

\section{ACKNOWLEDGMENTS}

This work was supported by the Institut National d'Astronomie et de Géophysique under grant No. 83-070978, by the Commissariat a l'Energie Atomique under grant 1617 A4 and by the U.S. Department of Energy, BES-Materials Sciences, under Contract No. W-31-109-ENG-38. We wish to also thank J. M. Besson for critical reading of the manuscript. This work was performed during a visit of M.G. to the Laboratoire de Physique des Milieux Condensés.

'A. Jayaraman, Rev. Mod. Phys. 55, 65 (1983).

${ }^{2}$ A. Polian, J. M. Besson, M. Grimsditch, and H. Vogt, Phys. Rev. B 25, 2767 (1982).

${ }^{3}$ K. Strossner, S. Ves, and M. Cardona, Phys. Rev. B 32, 6614 (1985).

${ }^{4}$ H. Shimizu, E. M. Brody, H. K. Mao, and P. M. Bell, Phys. Rev. Lett. 47, 128 (1981).

${ }^{5}$ J. P. Itie and R. Letoullec, J. Phys. (Paris) 45, C8-53 (1984).

${ }^{6} \mathrm{~J}$. van Straaten, R. J. Wijngaarden, and I. F. Silvera, Phys. Rev. Lett. 48, 97 (1982).

${ }^{7}$ R. M. Hazen, H. K. Mao, L. W. Finger, and P. M. Bell, Carnegie Inst. Washington Year. 79, 348 (1980); G. Zou, H. K. Mao, and P. M. Bell, Carnegie Inst. Washington Year. 81, 392 (1982); J. Xu, H. K. Mao, and P. M. Bell, High Temp. High Pressures 16, 495 (1984).

${ }^{\mathrm{k}} \mathrm{G}$. J. Piermarini, S. Block, J. D. Barnett, and R. A. Forman, J. Appl. Phys. 46, 2774 (1975).

${ }^{9}$ M. Grimsditch, P. Loubeyre, and A. Polian, Phys. Rev. B 33, 7192 (1986).

${ }^{10} M$. Lallemand and D. Vidal, J. Chem. Phys. 66, 4776 (1976).

"H. K. Mao and P. M. Bell, Carnegie Inst. Washington Year. 76, 646 (1977).

${ }^{12}$ M. Grimsditch and A. Polian (unpublished). 